\title{
Transverse Mode Switching and Locking in Vertical-Cavity Surface-Emitting Lasers Subject to Orthogonal Optical Injection
}

\author{
Angel Valle, Ignace Gatare, Krassimir Panajotov, and Marc Sciamanna
}

\begin{abstract}
In this paper, we report on theoretical and experimental investigation on polarization and transverse mode behavior of vertical-cavity surface-emitting lasers (VCSELs) under orthogonal optical injection as a function of the injection strength and of the detuning between the injection frequency and the free-running frequency of the solitary laser. As the injection strength increases the VCSEL switches to the master laser polarization. We find that the injection power necessary to obtain such polarization switching is minimum at two different values of the frequency detuning: the first one corresponds to the frequency splitting between the two linearly polarized fundamental transverse modes, and the second one appears at a larger positive frequency detuning, close to the frequency difference between the first-order and the fundamental transverse modes of the solitary VCSEL. We show theoretically that both the depth and the frequency corresponding to the second minimum increase when the relative losses between the two transverse modes decrease. Bistability of the polarization switching is obtained for the whole frequency detuning range. Such a bistability is found for the fundamental mode only or for both transverse modes, depending on the value of the detuning. The theoretical and experimental optical spectra are in good agreement showing that the first-order transverse mode appears locked to the external injection.
\end{abstract}

Index Terms-Injection locking, nonlinear dynamics, polarization switching, transverse modes, vertical-cavity surface-emitting laser (VCSEL).

\section{INTRODUCTION}

O PTICAL injection in semiconductor lasers is a research topic that has attracted much interest since the early 1980s [1], [2]. When injection locking is achieved, several

Manuscript received November 2, 2006; revised December 21, 2006. This work was supported in part by the IAP "Photon" Network of the Belgian government, in part by FWO-Flanders, GOA, and OZR of the Vrije Universiteit Belgium, Brussels, Belgium, and in part by COST 288 European Action. The work of A.Valle was supported in part by the Comisión Interministerial de Ciencia y Tecnología (CICYT) under Project TEC2006-13887-C05-02/TCM and from Gobierno de Cantabria under Project UCAN05-23-012.

A. Valle is with the Instituto de Física de Cantabria, Consejo Superior de Investigaciones Cientificas (CSIC), Universidad de Cantabria, E-39005 Santander, Spain (e-mail: valle@ifca.unican.es).

I. Gatare is with the Ecole Supérieure d'Electricité (Supélec), Laboratoire Matériaux Optiques, Photonique et Systèmes (LMOPS), Centre National de la Recherche Scientifique (CNRS), F-57070 Metz, France and also with the Department of Applied Physics and Photonics (TW-TONA), Vrije Universiteit Brussels, B-1050 Brussels, Belgium (e-mail: gatarega ign@metz.supelec.fr).

K. Panajotov is with the Department of Applied Physics and Photonics (TW-TONA), Vrije Universiteit Brussels, B-1050 Brussels, Belgium and also with the Institute of Solid State Physics, 1784 Sofia, Bulgaria (e-mail: kpanajotov@tona.vub.ac.be).

M. Sciamanna is with the Ecole Supérieure d'Electricité (Supélec), Laboratoire Matériaux Optiques, Photonique et Systèmes (LMOPS), Centre National de la Recherche Scientifique (CNRS), F-57070 Metz, France (e-mail: marc.sciamanna@supelec.fr).

Digital Object Identifier 10.1109/JQE.2007.893004 improvements of the injected semiconductor laser characteristics are obtained like laser spectral narrowing [3], suppression of laser noise [4], reduction of frequency chirp under modulation [5], and improvement of the laser intrinsic frequency response [6]. Recent applications of optical injection include clock recovery by injection locking of passively mode-locked semiconductor lasers [7], waveform reshaping based on the transition between unlocked and locked laser state [8], optical generation of millimeter waves with low phase noise for the use in radio-over-fiber (RoF) systems [9], [10], optical injection phase-locked loop (OIPLL) for synchronization of lasers [11], the development of light sources with reduced nonlinear distortion and improved spurious-free dynamic range (SFDR) for the use in analog fiber optic links [12]. Semiconductor lasers can be divided in two main categories depending on the dimensions and the geometry of the active cavity: edge-emitting lasers (EEL) and vertical-cavity surface-emitting lasers (VCSELs). VCSELs present significant advantages over their edge-emitting counterparts, including low threshold current, low cost, circular output beam, and easy fabrication in two-dimensional arrays. Although VCSELs are intrinsically single-longitudinal mode devices, emission in multiple transverse and polarization modes is usually found [13]. The polarization is not well fixed and small changes of the injection current or the device temperature may result in a polarization switching (PS) between the two linearly polarized modes. While emission in several transverse modes is usually attributed to (SHB) effects [13]-[15], a number of different physical mechanisms can be responsible for PS phenomenon in VCSELs. Therefore, different models of PS in VCSELs have been suggested, for example those taking into account spin relaxation mechanisms in semiconductor quantum wells spin flip model (SFM) [16], [17], thermal effects [18], or the relative modification of the net modal gain and losses with the injection current [19]-[21].

VCSELs are also attractive for use in injection locking because of its compactness, low power consumption, and circular output beam [22], [23]. Besides its fundamental research interest, optical injection in VCSELs can be used in all-optical and reconfigurable optical switches [24], to significatively increase the resonance frequency in VCSELs [22], to achieve transverse mode selection [25], or to perform several optical signal processing functions [26]. Stable injection locking within a large frequency detuning range has been observed when both the VCSEL and the injected light polarizations were parallel [27]. In that configuration, optical injection can be used to obtain chaotic instabilities in VCSELs [28]. A different configuration 
was used in the seminal experimental work by Pan et al. [29]: linearly polarized light in the vertical polarization was injected from an external laser source in a VCSEL which current was fixed in such a way that it emitted in a single mode with horizontal polarization. We shall call this configuration "orthogonal optical injection." It was found that PS can be achieved through injection locking for certain values of wavelength detunings and injected power. A recent experimental study [30], [31] performed over a wider frequency detuning range (from -82 to $89 \mathrm{GHz}$ ) revealed different regions of qualitatively different dynamical behaviors: a region of frequency locking, a region of PS with bistability, wave mixing, subharmonic resonance, time-periodic, and chaotic dynamics. Those different behaviors were summarized in a mapping of the bifurcation boundaries in the plane of the injection parameters: injection power $P_{\mathrm{inj}}$ and frequency detuning between the external "master" laser (ML) and the injected "slave" laser (SL), $\Delta \nu=\nu_{\mathrm{ML}}-\nu_{\mathrm{SL}}$. That diagram was characterized by the existence of a minimum injection power needed to obtain PS, that appeared at a detuning corresponding to the frequency splitting between the two linearly polarized fundamental modes of the VCSEL. Similar results have been theoretically obtained by using a model for the two linear polarizations of the fundamental transverse mode of a VCSEL [32], [33].

In this work, the output characteristics of an oxide-confined AlGaAs-As quantum-well VCSEL under orthogonal optical injection have been mapped as a function of the optical injection strength and the frequency detuning between MLs and SLs. This work extends the previous measurements [30] over larger frequency detunings (up to $180 \mathrm{GHz}$ ). We find that a new minimum of the injection power needed to obtain PS appears at a positive detuning of around $150 \mathrm{GHz}$. This frequency detuning is near the frequency difference between the fundamental and the first-order transverse modes of the solitary VCSEL. The first-order transverse mode is then playing a key role in the creation of this new minimum. This has motivated us to perform a theoretical study by using a model that includes polarized multitransverse mode operation. We show that the frequency detuning at which the second minimum appears is indeed mainly determined by the frequency splitting between the fundamental and the first-order transverse modes. We find that the depth and the frequency position of that minimum increase as the relative losses between the two transverse modes decrease, in agreement with our experimental results. Our theoretical and experimental results also show that a bistability in PS is obtained for the whole frequency detuning range. Such a bistability is obtained for the fundamental mode only or for both transverse modes, depending on the value of the detuning. The theoretical optical spectra reveal that the first-order transverse mode appears locked to the external injection, also in an agreement with our experimental results.

Our paper is organized as follows. In Section II, the theoretical model is presented. In Section III, we present our theoretical results corresponding to an orthogonal optical injection in multitransverse mode VCSELs. In Section IV-A, we describe our experimental setup. In Section IV-B, we present the experimental results showing PS and transverse mode competition induced by the orthogonal optical injection. Finally, in Section V,
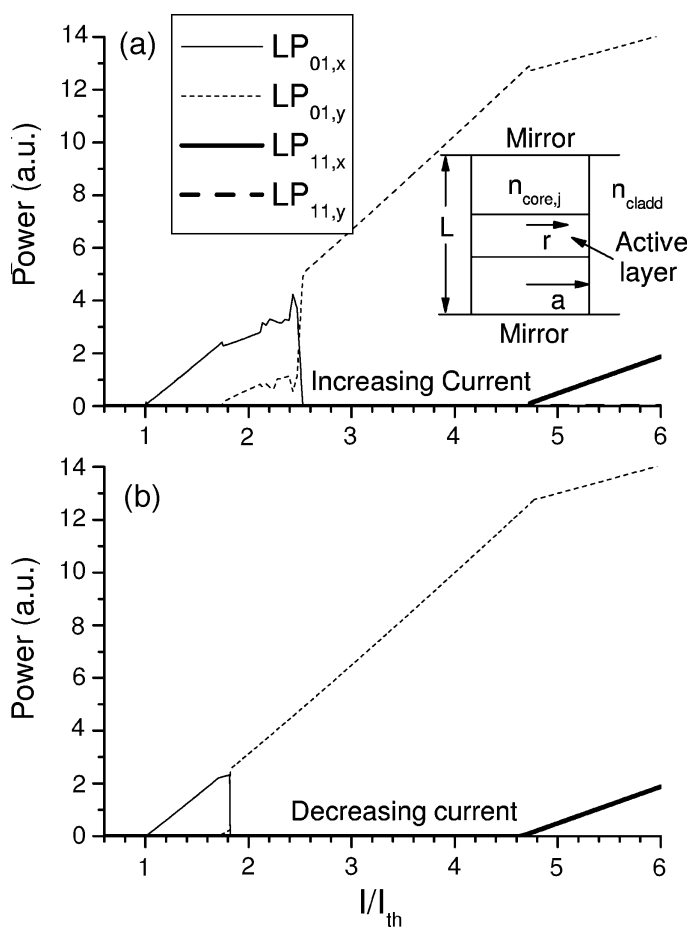

Fig. 1. (a) Light-current characteristics of a multimode VCSEL without optical injection. This figure is obtained by fixing $k_{i j}=0, \kappa_{r}=1.2$, and by increasing linearly the value of the current $I$ from $0.1 I_{\mathrm{th}}$ to $6 I_{\mathrm{th}}$ during $10 \mu \mathrm{s}$. (b) $L-I$ characteristics when decreasing the current from $6 I_{\text {th }}$ to $0.1 I_{\text {th }}$ during $10 \mu \mathrm{s}$. The inset of Fig. 1 shows an schematic diagram of the VCSEL.

a discussion on the agreement between theoretical and experimental results and a summary are presented.

\section{MODEL}

In this work, we consider a model [34], [35] that takes into account two of the mechanisms that can define the polarization of a VCSEL. The first one is associated with the combined effect of the VCSEL anisotropies, the linewidth enhancement factor and the spin-flip relaxation processes within a framework known as the SFM model [16]. The second mechanism is related to the effect of having different electrical field profiles for each linear polarization due to the birefringence of the device [36]. We consider cylindrically symmetric weak index-guided devices with the structure illustrated in the inset of Fig. 1(a). The radius of the core region and the length of the cavity are denoted as $a$ and $L$, respectively. Subscripts $x$ and $y$ will be used to denote the polarization direction. Birefringency is taken into account by assuming that the core refractive index in the $x$ direction, $n_{\text {core }, x}$, is larger than in the $y$ direction, $n_{\text {core }, y}$, hence, the $x$ polarized mode emission frequency is lower than that of the $y$ polarized mode, while the cladding refractive index, $n_{\text {cladd }}$, is the same in both directions. We will consider a small value of the index step (0.011) in such a way that the appropriate transverse modes of the structure are the $\mathrm{LP}_{\mathrm{mn}}$ modes. That index step is greater than the contribution due to the carrier-induced refractive index [37] and then the evolution obtained with our model, based on a modal expansion, coincides with the one obtained with a full spatiotemporal model [37]. Here we treat the case of VCSELs that can operate in the fundamental $\left(\mathrm{LP}_{01}\right)$ and in the first-order $\left(\mathrm{LP}_{11}\right)$ transverse modes. Subscripts 0,1 will be used to denote 
the $\mathrm{LP}_{01}$ and $\mathrm{LP}_{11}$ modes, respectively. In the basis of the linearly polarized modes and considering radial symmetry of the cavity the optical field can be written as [34], [35]

$$
\begin{aligned}
\vec{E}(r, t)= & {\left[\left(E_{0 x}(t) \psi_{0 x}(r)+E_{1 x}(t) \psi_{1 x}(r)\right) \vec{x}\right.} \\
& \left.+\left(E_{0 y}(t) \psi_{0 y}(r)+E_{1 y}(t) \psi_{1 y}(r)\right) \vec{y}\right] e^{i k \alpha t}+\text { c.c. }
\end{aligned}
$$

where $\psi_{0 j}$ and $\psi_{1 j}$ are the modal profiles of the $\mathrm{LP}_{01}$ and $\mathrm{LP}_{11}$ modes, respectively, obtained by solving the Helmholtz equation [34]; $E_{0 j}$ and $E_{1 j}$ are the modal amplitudes of these modes; the subindex $j$ stands for the linear polarization state of the given mode; $k$ is the electric field decay rate that includes the internal and facet losses [2]; and $\alpha$ is the alpha factor or linewidth enhacement factor that describes phase-amplitude coupling mechanisms in semiconductor lasers [2]. The equations describing the polarization and transverse mode behavior of the VCSEL with an injected optical field, written appropriately in the cylindrical basis, read [35]

$$
\begin{aligned}
& \dot{E}_{0 x}=k(1+i \alpha)\left(E_{0 x}\left(g_{0 x}-1\right)+i E_{0 y} g_{0 x y}\right) \\
& -\left(\gamma_{a}+i \gamma_{p 0}\right) E_{0 x}+\frac{k_{0 x}}{\tau_{\text {in }}} e^{i \Delta \omega t} \\
& +\sqrt{\frac{\beta}{2}}\left(\sqrt{\bar{N}+\bar{n}} \xi_{0+}(t)+\sqrt{\bar{N}-\bar{n}} \xi_{0-}(t)\right) \\
& \dot{E}_{0 y}=k(1+i \alpha)\left(E_{0 y}\left(g_{0 y}-1\right)-i E_{0 x} g_{0 y x}\right) \\
& +\left(\gamma_{a}+i \gamma_{p 0}\right) E_{0 y}+\frac{k_{0 y}}{\tau_{\text {in }}} e^{i \Delta \omega t} \\
& -i \sqrt{\frac{\beta}{2}}\left(\sqrt{\bar{N}+\bar{n}} \xi_{0+}(t)-\sqrt{\bar{N}-\bar{n}} \xi_{0-}(t)\right) \\
& \dot{E}_{1 x}=k(1+i \alpha)\left(E_{1 x}\left(g_{1 x}-\kappa_{r}\right)+i E_{1 y} g_{1 x y}\right) \\
& +i \gamma_{p}^{t r} E_{1 x}-\left(\gamma_{a}+i \gamma_{p 1}\right) E_{1 x}+\frac{k_{1 x}}{\tau_{\text {in }}} e^{i \Delta \omega t} \\
& +\sqrt{\frac{\beta}{2}}\left(\sqrt{\bar{N}+\bar{n}} \xi_{1+}(t)+\sqrt{\bar{N}-\bar{n}} \xi_{1-}(t)\right) \\
& \dot{E}_{1 y}=k(1+i \alpha)\left(E_{1 y}\left(g_{1 y}-\kappa_{r}\right)-i E_{1 x} g_{1 y x}\right) \\
& +i \gamma_{p}^{t r} E_{1 y}+\left(\gamma_{a}+i \gamma_{p 1}\right) E_{1 y}+\frac{k_{1 y}}{\tau_{\text {in }}} e^{i \Delta \omega t} \\
& -i \sqrt{\frac{\beta}{2}}\left(\sqrt{\bar{N}+\bar{n}} \xi_{1+}(t)-\sqrt{\bar{N}-\bar{n}} \xi_{1-}(t)\right) \\
& \frac{\partial N(r, t)}{\partial t}=I(r)+D \nabla_{\perp}^{2} N-\gamma_{e} \\
& \times\left[N\left(1+\sum_{i=0,1} \sum_{j=x, y}\left|E_{i j}\right|^{2} \psi_{i j}^{2}(r)\right)\right. \\
& \left.-i n \sum_{i=0,1}\left(E_{i x} E_{i y}^{*}-E_{i y} E_{i x}^{*}\right) \psi_{i x}(r) \psi_{i y}(r)\right] \\
& \frac{\partial n(r, t)}{\partial t}=-\gamma_{s} n+D \nabla_{\perp}^{2} n-\gamma_{e} \\
& \times\left[n \sum_{i=0,1} \sum_{j=x, y}\left|E_{i j}\right|^{2} \psi_{i j}^{2}(r)\right. \\
& \left.-i N \sum_{i=0,1}\left(E_{i x} E_{i y}^{*}-E_{i y} E_{i x}^{*}\right) \times \psi_{i x}(r) \psi_{i y}(r)\right]
\end{aligned}
$$

TABLE I

PARAMETERS USED FOR THE SIMULATION OF THE VCSEL

\begin{tabular}{ccc}
\hline SYMBOL & VALUE & MEANING OF THE SYMBOL \\
\hline$a$ & $3 \mu \mathrm{m}$ & Radius of the core region \\
$L$ & $1 \mu \mathrm{m}$ & Length of the cavity \\
$n_{\text {core }, x}$ & 3.50002084 & Refractive index of the core in the x-direction \\
$n_{\text {core } y}$ & 3.5 & Refractive index of the core in the y-direction \\
$n_{\text {cladd }}$ & 3.489 & Refractive index of the cladding region \\
$k$ & $300 \mathrm{~ns}^{-1}$ & Field decay rate \\
$\alpha$ & 3 & Linewidth enhacement factor \\
$\gamma_{e}$ & $0.55 \mathrm{~ns}^{-1}$ & Decay rate for the total carrier population \\
$\gamma_{s}$ & $91 \mathrm{~ns}^{-1}$ & Spin-flip relaxation rate \\
$D$ & $10 \mathrm{~cm}^{-1} \mathrm{~s}^{-1}$ & Diffusion coefficient \\
$\beta$ & $10^{-1} \mathrm{~s}^{-1}$ & Spontaneous emission coefficient \\
$\gamma_{a}$ & $-0.81 \mathrm{~ns}^{-1}$ & Dichroism \\
$\eta_{i n j}$ & 1 & Coupling efficiency \\
$R$ & 0.995 & Output-mirror reflectivity \\
\hline
\end{tabular}

where $N(r, t)$ is the total carrier number and $n(r, t)$ is the difference in the carrier numbers of the two magnetic sublevels. $\kappa_{r}$ is the relative loss of the $\mathrm{LP}_{11}$ mode with respect to the $\mathrm{LP}_{01}$ mode. That parameter determines the value of the injection current at which the $\mathrm{LP}_{11}$ mode begins lasing. $I(r)$ represents a uniform current injection of over a circular disk of $3-\mu \mathrm{m}$ radius, and then $I(r)=I$ if $r<3 \mu \mathrm{m}$, and $I(r)=0$, elsewhere. The normal gain normalized to the threshold gain, $g_{i j}(i=0,1$, $j=x, y$ ), is defined as

$$
g_{i j}=\frac{\int_{0}^{\infty} N(r, t) \psi_{i j}^{2}(r) r d r}{\int_{0}^{\infty} \psi_{i j}^{2}(r) r d r}
$$

and $g_{i j k}(i=0,1 ; j k=x y, y x)$ is given by

$$
g_{i, j k}=\frac{\int_{0}^{\infty} n(r, t) \psi_{i j}(r) \psi_{i k}(r) r d r}{\int_{0}^{\infty} \psi_{i j}^{2}(r) r d r} .
$$

Note that the modal gains for the $x$ and $y$ polarizations are different due to the different optical mode profiles. However, we neglect the material gain difference since the frequency splitting is very small compared to the width of the gain curve. The injection terms are characterized by the $k_{i j}$ injection strengths, the VCSEL round-trip time, $\tau_{\text {in }}=2 L / v_{g}$, where $v_{g}$ is the group velocity, and the detuning $\Delta \omega=\omega_{\text {inj }}-\omega_{\text {th }}$, where $\omega_{\text {inj }}$ is the frequency of the ML and $\omega_{\text {th }}=\left(\omega_{0 x}+\omega_{0 y}\right) / 2$, is the central frequency between the two polarizations of the fundamental mode. The injection strength $k_{i j}$ is given by

$$
k_{i j}=\left(\frac{1}{\sqrt{R}}-\sqrt{R}\right) \sqrt{\eta_{\mathrm{inj}}} \sqrt{P_{\mathrm{inj}, i j}}
$$

where $R$ is the output-mirror reflectivity, $\eta_{\text {inj }}$ is the coupling efficiency of the injected light to the optical field in the laser cavity, and $P_{\mathrm{inj}, i j}$ is the power injected in the $j$-polarization of the $i$-transverse mode [2]. The rest of the parameters that appear in the equations are specified in the Table I. The frequency splitting between the orthogonal polarizations of the $\mathrm{LP}_{01}$ mode, $2 \gamma_{p 0} /(2 \pi)$, between the orthogonal polarizations of the $\mathrm{LP}_{11}$ mode, $2 \gamma_{p 1} /(2 \pi)$, and between the two transverse modes with the same polarization, $\gamma_{p}^{t r} /(2 \pi)$, are obtained from the calculation of the waveguide modes via the Helmholtz equation. We have chosen the values of $n_{\text {core }, x}, n_{\text {core }, y}$ and $n_{\text {cladd }}$ in such a way that $2 \gamma_{p 0} /(2 \pi)=2 \mathrm{GHz}$ and $\gamma_{p}^{t r} /(2 \pi)=193 \mathrm{GHz}$, that correspond to the experimental values found for the solitary VCSEL of Section IV. Spontaneous emission noise processes are modeled 
by the terms $\xi_{ \pm}$taken as complex Gaussian white noise sources of zero mean and delta-correlated in time. In the noise terms, the carrier distribution is integrated over the active region

$$
\bar{N}=\frac{\int_{0}^{a} N(r, t) r d r}{a^{2}}, \quad \bar{n}=\frac{\int_{0}^{a} n(r, t) r d r}{a^{2}} .
$$

In the following section, we will present the results obtained by integrating numerically the previous set of equations. Time and space integration steps of $0.01 \mathrm{ps}$ and $0.12 \mu \mathrm{m}$, respectively, have been used. The boundary conditions for the carrier distribution are taken as $N(\infty, t)=0, n(\infty, t)=0$. The initial conditions correspond to the below threshold stationary solution, i.e to $I=0.1 I_{\mathrm{th}}$, where $I_{\mathrm{th}}$ is the threshold current.

\section{THEORETICAL RESUlTS}

We first present some results corresponding to the solitary VCSEL operation. The transverse and polarization mode-resolved light-current characteristics of the solitary VCSEL is shown in Fig. 1(a). The VCSEL begins to emit in the fundamental mode with the smallest frequency, $\mathrm{LP}_{01, x}$. A type II PS, i.e., from the lower to the higher frequency, within the fundamental mode is obtained at around 2.5 times the threshold current. The VCSEL then emits in the $\mathrm{LP}_{01, y}$ mode until the higher order mode with orthogonal polarization appears at around $4.7 I_{\mathrm{th}}$. The value of the relative losses of the $\mathrm{LP}_{11}$ with respect to the $\mathrm{LP}_{01}$ mode has been chosen $\left(\kappa_{r}=1.2\right)$ in such a way that the $\mathrm{LP}_{11}$ mode appears near the experimental value found for the solitary VCSEL (see Section IV). In Fig. 1(b), we also show the light-current characteristics obtained when decreasing the current in the same way. The VCSEL switches back to the $x$ direction at $1.83 I_{\mathrm{th}}$, hence demonstrating a hysteresis zone.

We now consider the effect of the orthogonal optical injection on the transverse modes and polarization behavior of the VCSEL. We first set the current at a value, $I=1.7 I_{\mathrm{th}}$, slightly smaller than the lowest current of the hysteresis region of the solitary VCSEL. Orthogonal optical injection is then modeled by choosing $k_{i x}=0$ and $k_{i y}>0$ since at that value of the current the VCSEL emits in the $x$ polarization of the $\mathrm{LP}_{01}$ mode. We show in Fig. 2 the boundaries of PS in the plane of injection parameters (the detuning and the injected power). The injected power has been normalized to the output power of the solitary VCSEL and has been taken in logarithmic scale. For each value of the frequency detuning we have performed a sweeping along the horizontal axis, that is, increasing and then decreasing the injected power. That sweeping is performed in the following way: we first let the solitary VCSEL to reach the steady state and then we change the injected power step by step. We consider 100 steps between the minimum and the maximum value of the injected power. We calculate the average of each polarized transverse mode over the last nanosecond of each step (of 2-ns duration). We consider that a polarization switch-on (switch-off) is obtained when the averaged total $y$-polarized power becomes larger (smaller) than the averaged total $x$-polarized power. The
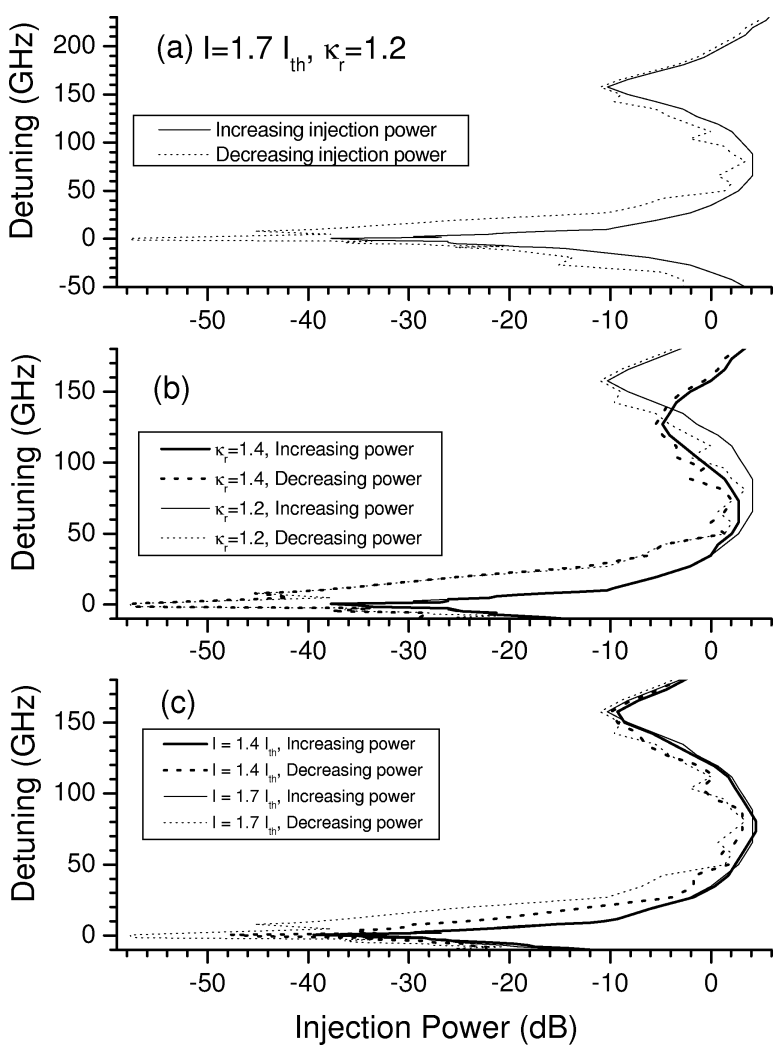

Fig. 2. Injection power required for PS in a VCSEL subject to orthogonal optical injection. Switching from $x$ to $y$ ( $y$ to $x$ ) polarization when increasing (decreasing) the injection power is shown with solid (dotted) lines. (a) Corresponds to $I=1.7 I_{\text {th }}$ and $\kappa_{r}=1.2$. (b) Results for $I=1.7 I_{\text {th }}$ and $\kappa_{r}=1.4$. (c) Rresults for $I=1.4 I_{\mathrm{th}}$ and $\kappa_{r}=1.2$.

polarization switch-on (switch-off) points for increasing (decreasing) the power of the injected light are represented by solid (dotted) lines in Fig. 2, respectively. In Fig. 2(a), the results for the case of $I=1.7 I_{\mathrm{th}}$ and $\kappa_{r}=1.2$ show that there are two minima in the injected power required for switching. Those minima appear at around 0 and $155 \mathrm{GHz}$ for both the cases of increasing and decreasing the injection power. The detuning at which the second minimum appears is near the frequency difference between the $\mathrm{LP}_{01}$ and the $\mathrm{LP}_{11}$ transverse modes of the solitary VCSEL. The injection power for the PS-off is smaller than the one required for the PS-on and then a region of bistable operation of the device is obtained. Bistable operation is obtained over wider ranges of injection powers when the detuning is near the $0 \mathrm{GHz}$ minimum.

The effect of the transverse mode dichroism on the mapping of PS is analyzed in Fig. 2(b). In that figure, we compare the results obtained with the previously described VCSEL $\left(\kappa_{r}=\right.$ 1.2) with another VCSEL in which the higher order modes are much more suppresed $\left(\kappa_{r}=1.4\right)$; in fact, the first higher order mode appears at $I=17.5 I_{\text {th }}$ when $\kappa_{r}=1.4$. The injected power required for PS keeps on having two minima. The PS boundaries do not change in a significant way if the detuning is smaller than $35 \mathrm{GHz}$. For higher values of the detuning, there are several changes: the minimum injected power required for switching increases and the detuning at which this minimum appears decreases to $127 \mathrm{GHz}$. The effect of the injection current 

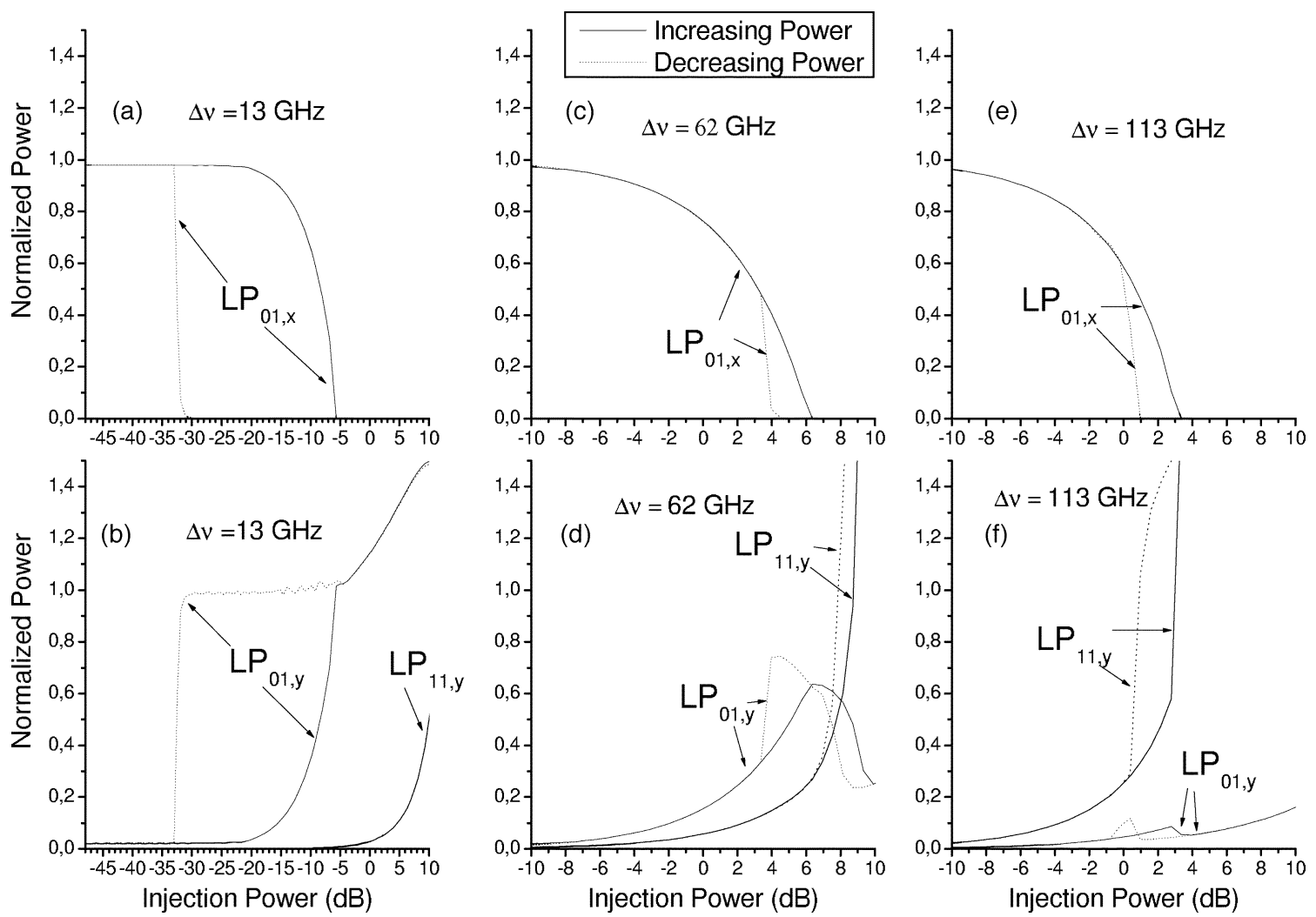

Fig. 3. Averaged powers of the polarized transverse modes as a function of the injection power. The upper (lower) part of the figure corresponds to $x$ - ( $y$-) polarized light. First, second, and third columns correspond to 13, 62, and $113 \mathrm{GHz}$ frequency detunings, respectively. Results obtained when increasing (decreasing) the injection power are plotted with solid (dotted) lines.

is also analyzed in Fig. 2(c). In that figure, we have decreased the injection current applied to the VCSEL of Fig. 2(a) to a value that is clearly below the hysteresis region, $I=1.4 I_{\mathrm{th}}$. No appreciable effects are observed for the switch-on curve when changing the injection current. However, the switch-off curve changes in such a way that the width of the hysteresis region decreases when decreasing the injection current.

The interpretation of some of the previous results can be obtained with the help of Figs. 3-5. In Fig. 3, the averaged power of the different transverse modes is plotted as a function of the injected power for three representative values of the frequency detuning: 13, 62, and $113 \mathrm{GHz}$. The upper (lower) part of the figure corresponds to $x$ - $(y$-) polarized power. Those averaged powers are normalized to the power of the solitary VCSEL. The results corresponding to a frequency detuning of $13 \mathrm{GHz}$, slightly larger than the one of the first minimum, are shown in Fig. 3(a) and (b). The solitary VCSEL is mainly emitting in the $\mathrm{LP}_{01, x}$ mode. We first analyze the behavior obtained when increasing the injection power. When the injection power is less than $-23 \mathrm{~dB}$, no appreciable changes are observed in the averaged modal powers. However, some changes are apparent from the optical spectra. Different optical spectra corresponding to representative values of the injection power when the detuning is $13 \mathrm{GHz}$ are shown in Fig. 4. Fig. 4(a) shows that a contribution to the $y$-polarized spectrum at the injection frequency appears for small values of the injection power. That contribution increases when the injection power increases. However the $x$-polarized spectrum is very similar to the corresponding solitary spectrum. When the injection power increases beyond $-23 \mathrm{~dB}$, the $\mathrm{LP}_{01, x}$ averaged power begins to decrease while the one of the $\mathrm{LP}_{01, y}$ begins to increase, as seen in Fig. 3(a) and (b). Fig. 4(b) shows that the increase of the $\mathrm{LP}_{01, y}$ power appears mainly at the injection frequency. Wave mixing is also observed by the peaks in the $x$ - and $y$ polarization appearing at multiples of the frequency difference $\nu_{M}-\nu_{01, x}$ between the ML frequency and the VCSEL LP $\mathrm{P}_{01, x}$ mode. PS within the fundamental mode appears at $-8 \mathrm{~dB}$ and is illustrated in Fig. 3(a) and (b) and Fig. 4(c). After the PS, the VCSEL emits at a several frequencies at multiples of $\nu_{M}-\nu_{01, x}$. Fig. 3(b) also shows that further increase of the injected power leads to the excitation of the $\mathrm{LP}_{11, y}$ mode as well.

A typical optical spectrum in this regime is the one shown in Fig. 4(e) that corresponds to an injection power of $0 \mathrm{~dB}$. The optical spectrum is such that only one peak of the $y$-polarized light at the injection frequency appears, i.e., injection locking has been achieved. The previously described situation changes when decreasing the injection power, as it can be seen in Fig. 3(a) and (b): a wide bistability region appears for the $\mathrm{LP}_{01, x}$ and $\mathrm{LP}_{01, y}$ modes. However, no bistability is observed for the $\mathrm{LP}_{11, y}$ mode. A value of the injection power of $-20 \mathrm{~dB}$, in the middle of the hysteresis region has been chosen in Fig. 4(b) and (d) to illustrate the bistability by using optical spectra.

We now describe results for a detuning of $62 \mathrm{GHz}$ that corresponds to the local maximum of the injection power required for 

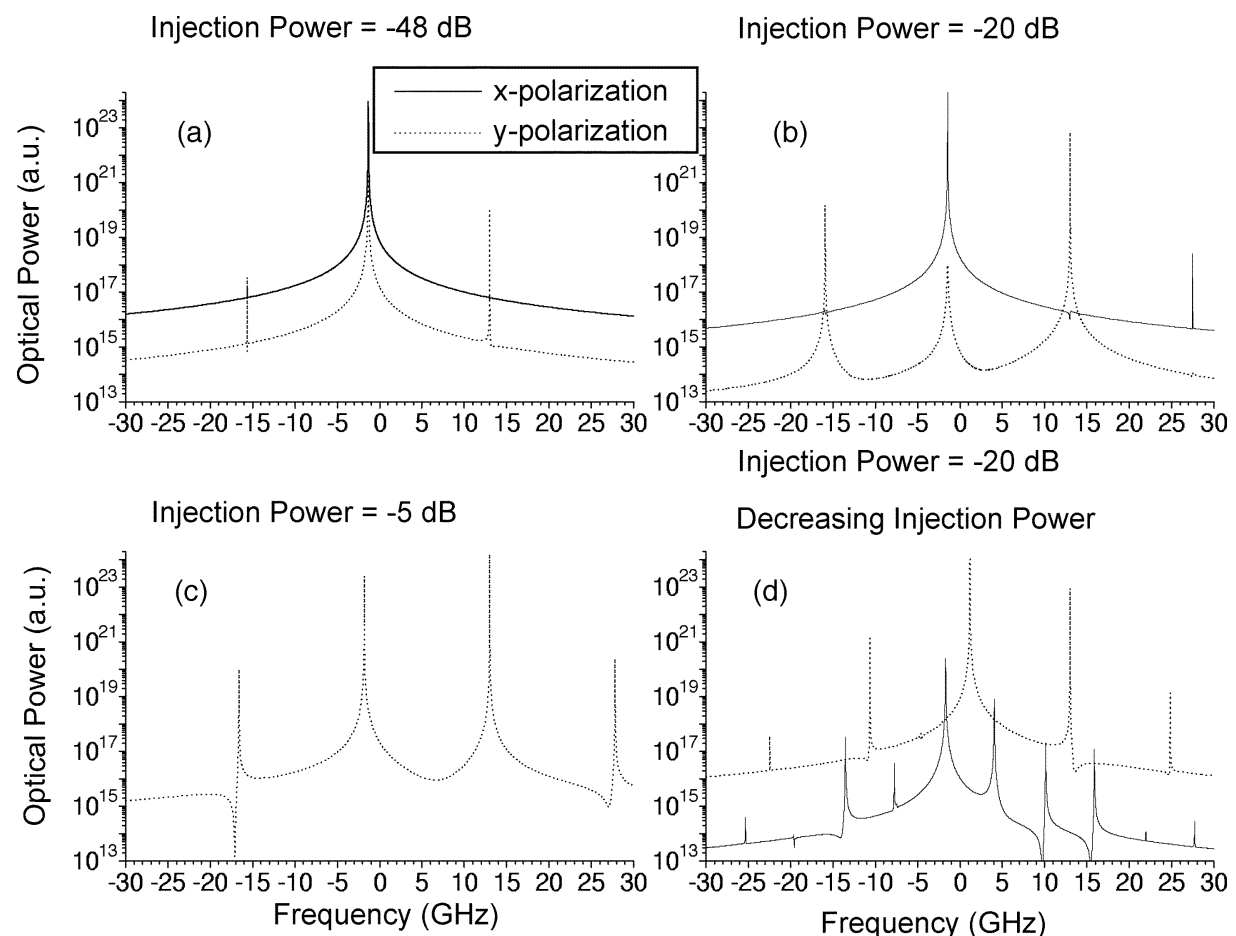

Decreasing Injection Power

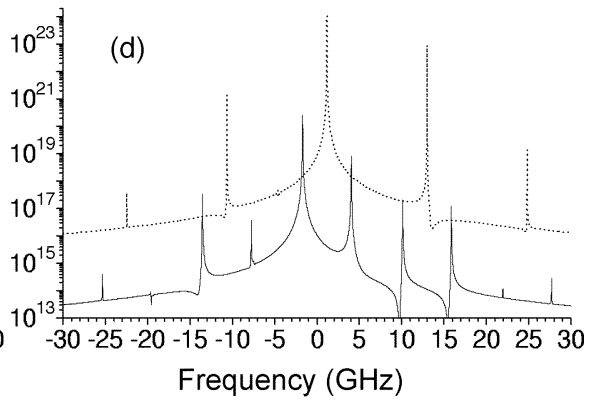

Injection Power $=0 \mathrm{~dB}$

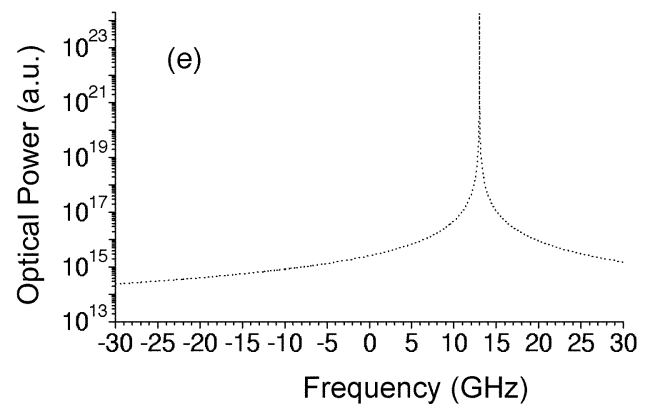

Fig. 4. Polarization resolved optical spectra for several values of the injected power when the frequency detuning is $13 \mathrm{GHz}$. $x$ - and $y$-polarized spectra are plotted with solid and dotted lines, respectively. (a), (b), (c), and (e) correspond to increasing injection power while (d) corresponds to decreasing power. Zero frequency corresponds to injection at the central frequency between the two polarizations of the fundamental mode.

the PS-on in the mapping in Fig. 2(a). We first analyze the behavior obtained when increasing the injection power. A monotonic decrease of the $\mathrm{LP}_{01, x}$ averaged power together with a monotonic increases of the $\mathrm{LP}_{01, y}$ and $\mathrm{LP}_{11, y}$ averaged powers are obtained, as it can be seen in Fig. 3(c) and (d). PS-on occurs at around 3.5-dB injection power. There is an appreciable contribution of the $\mathrm{LP}_{11, y}$ mode to that PS, in contrast to the $13-\mathrm{GHz}$ detuning case. We also observe that there is a value of the injection power, around $8 \mathrm{~dB}$, beyond which the main contribution to the optical power is given by the $\mathrm{LP}_{11, y}$ mode. The situation again changes when decreasing the injection power because bistability regions appears now for all the transverse modes with orthogonal polarization. The width of the bistable region of the $\mathrm{LP}_{01}$ modes is smaller than the one for the $13-\mathrm{GHz}$ detuning case. However, bistability also appears for the $\mathrm{LP}_{11, y}$ mode. Typical optical spectra for the 62-GHz detuning case are illustrated in Fig. 5. Again, the component near the zero frequency (at the injection frequency) of the $y$-polarized spectrum decreases (increases) when increasing the injection power as it can be seen in Fig. 5(a) and (c). Optical spectra at $6.3 \mathrm{~dB}$, beyond the PS point, are also illustrated in Fig. 5(b) and (d), for increasing and decreasing optical injection, respectively. Only one peak of the $y$-polarized light at the injection frequency appears in both figures. Comparison between those figures also illustrate the bistability regime because the $x$-polarized power has disappeared when decreasing the injection power.

The results obtained for a frequency detuning approaching the second minimum of Fig. 2(a) are shown in Fig. 3(e) and (f). Again, a PS appears at around $1 \mathrm{~dB}$ injection power but now the contribution of the $\mathrm{LP}_{01, y}$ power to that switching is small. In fact the switching is mainly performed between the $\mathrm{LP}_{01, x}$ and the $\mathrm{LP}_{11, y}$ modes. We also show that when the averaged power of $\mathrm{LP}_{01, x}$ vanishes the growth of the $\mathrm{LP}_{11, y}$ modes becomes much larger while there is a small range of injection power, around $3 \mathrm{~dB}$, where the $\mathrm{LP}_{01, y}$ averaged power decreases. Fig. 3(e) and (f) also shows that the qualitative behavior obtained when decreasing the injection power is similar to the one obtained for the $62-\mathrm{GHz}$ detuning case. 

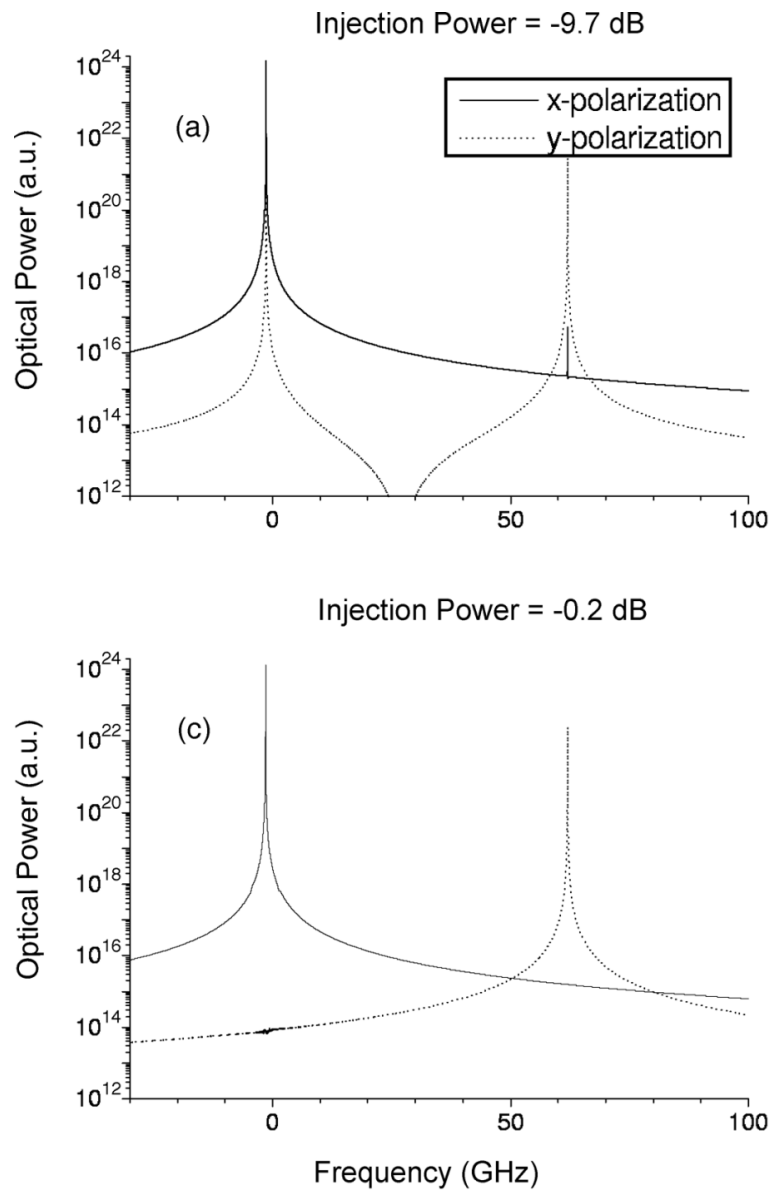

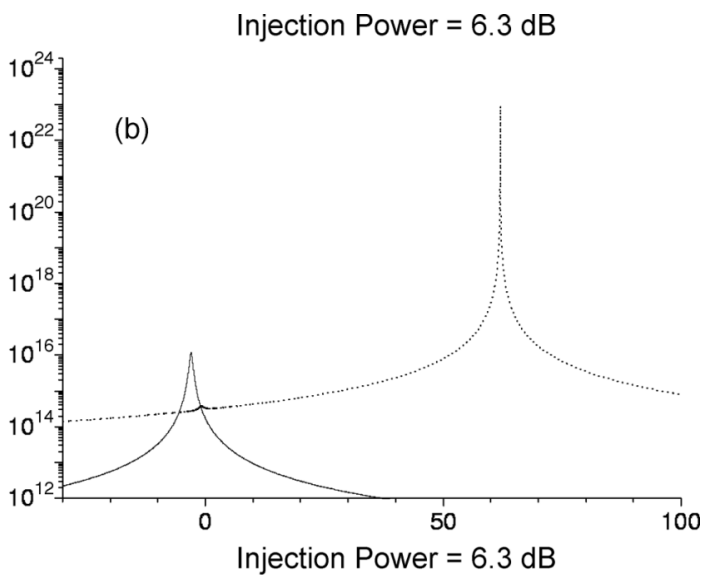

Decreasing Injection Power

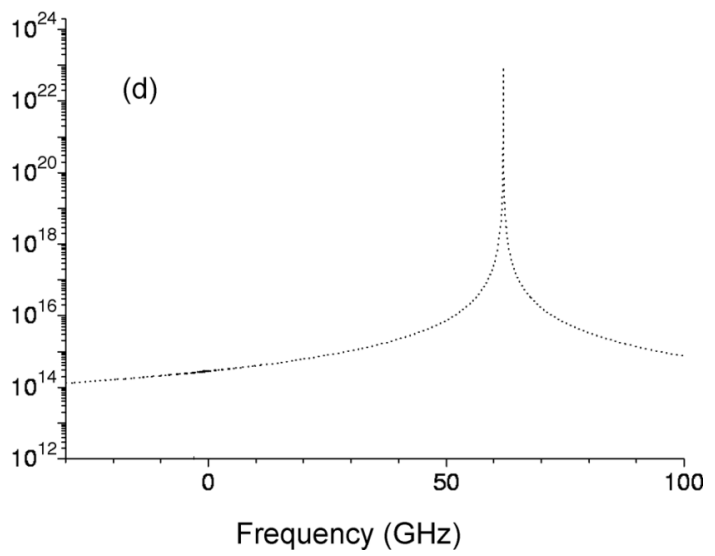

Fig. 5. Polarization resolved optical spectra for several values of the injected power when the frequency detuning is 62 GHz. $x$ and $y$-polarized spectra are plotted with solid and dotted lines, respectively. (a)-(c) correspond to increasing injection power while (d) corresponds to decreasing power.

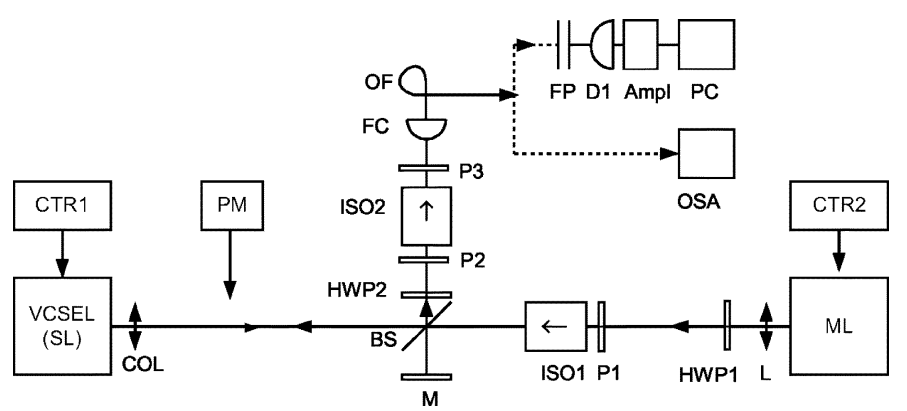

Fig. 6. Experimental setup of orthogonal optical injection in VCSEL. SL: slave laser; ML: master laser; COL: collimator; BS: beam splitter; HWP1-HWP2: half wave plate; ISO1; ISO2: optical isolators; L: lens; P1-P2: polarizers; M: mirror; FC: fiber coupling unit; OF: optical fiber; FP: Fabry-Pérot interferometer; D1: photodiode; Ampl: amplifier; PC: computer; OSA: optical spectrum analyzer; PM: power meter; CTR1 (CTR2): current driver and temperature controller of SL (ML). The VCSEL temperature was fixed at $20^{\circ} \mathrm{C}$.

\section{EXPERIMENTAL RESULTS}

\section{A. Experimental Setup}

Experimentally, the orthogonal optical injection is achieved using the setup presented in Fig. 6. A quantum-well VCSEL that emits around $845 \mathrm{~nm}$ is used as a SL. Its temperature and bias current are controlled by a low-noise laser driver (CTR1). An external-cavity laser diode is used as an ML. The wavelength of the light emitted by the ML can be tuned within the range of $845-855 \mathrm{~nm}$ by another low-noise laser driver (CTR2). The injection beam from the ML is then focused on the SL using a lens (L) while the light emitted by the SL is collimated by another lens (COL). An isolator (ISO1) with 36-40 dB of attenuation achieves a unidirectional coupling between the ML and the SL. The strength of the injected beam is varied using a polarizer P1. A half-wave plate (HWP1) fixes the polarization of the injected light to be orthogonal to the polarization direction of the free-running VCSEL. A nonpolarizing 50/50 beam-splitter and a mirror M are used to align the SL and the ML with the detection branch. The polarization in which the measurements are performed is selected by a half wave plate HWP2. The second isolator ISO2 with $36-40 \mathrm{~dB}$ of attenuation together with two polarizers P2 and P3 prevents the VCSEL from feedback-induced instabilities that may be generated by the light reflected by the fiber-coupling device FC. A power meter PM is used to measure the power emitted by the ML or the SL whereas spectral measurements are performed using either an optical spectrum analyzer (OSA) or a Fabry-Pérot spectrometer associated with a photodetector D1 and amplifier (Ampl) coupled to a computer (PC). 


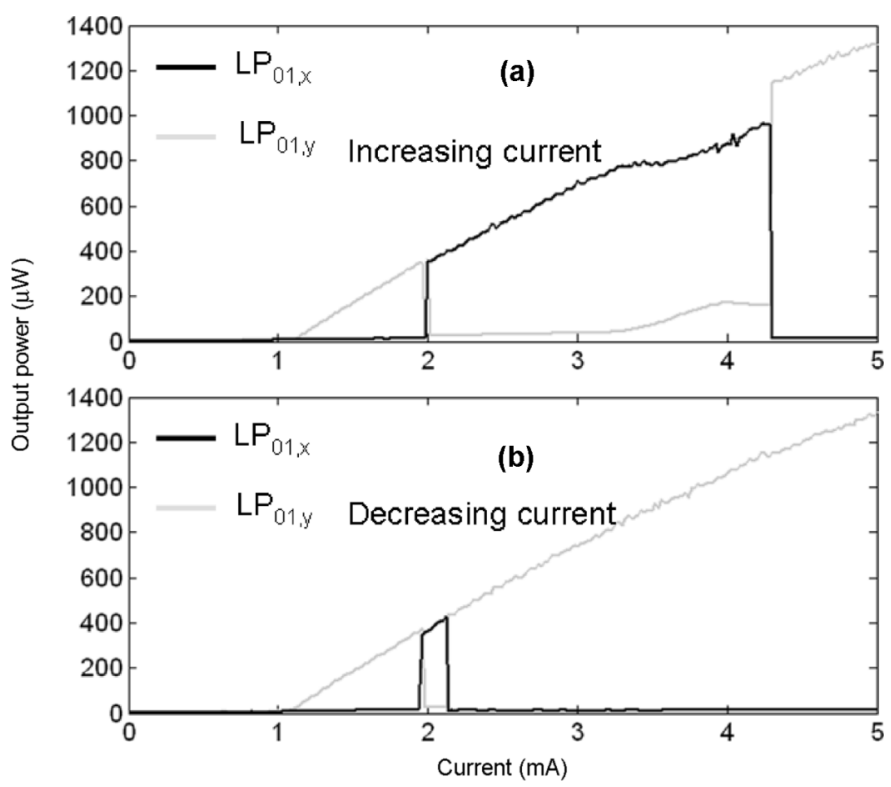

Fig. 7. Polarization-resolved light-current characteristics showing the evolution of the optical power in the horizontal (in black) and vertical (in gray) polarization direction. (a) VCSEL bias current is increased. (b) Bias current is decreased. The operating temperature is set at $20^{\circ} \mathrm{C}$.

\section{B. Experimental Results}

1) Light-Current Characteristics: The polarization-resolved light-current characteristic of the free-running VCSEL is presented in Fig. 7. If the bias current is increased [see Fig. 7(a)], the VCSEL first emits a fundamental linearly polarized vertical mode ( $y$-LP vertical mode) with a threshold current of $1 \mathrm{~mA}$. As the injection current is progressively increased, two successive PS between the orthogonal fundamental modes are found. The first switching (PS I) from the high-frequency mode ( $y$-LP mode) to the mode with a lower frequency ( $x$-LP mode) is observed for a bias current of $1.9 \mathrm{~mA}$. The second switching point (PS II) is found at a bias current of $4.2 \mathrm{~mA}$ and corresponds to PS from the $x$-LP horizontal mode to the $y$-LP vertical mode. Before PS II point is reached, we observe an elliptically polarized state which corresponds to a bump in the LI curve. However, if the VCSEL bias current is decreased after PS II has been achieved [see Fig. 7(b)], such an elliptical state is not found. As also shown in Fig. 7(b), a large hysteresis region is associated to PS II. In fact, if we decrease the injection current after PS II has been achieved, the VCSEL switches back to $x$-LP at a much lower current, i.e., $2.14 \mathrm{~mA}$. It is worth noting that the VCSEL exhibits a monomode operation for bias currents less than $5 \mathrm{~mA}$. For higher currents, excitation of the first-order transverse mode has been observed.

2) PS and Transverse Mode Competition: In the following section, the PS of the VCSEL with orthogonal optical injection is investigated experimentally in the plane of the injection parameters, i.e., in the frequency detuning versus injection power plane. The free-running VCSEL is biased at $2.105 \mathrm{~mA}$ [which is less than the lower limit of the hysteresis region associated to PS II-see Fig. 7(b)] and its temperature is stabilized at $20^{\circ} \mathrm{C}$. With these operating conditions, the free-running VCSEL emits a horizontal linearly polarized (LP) light. In

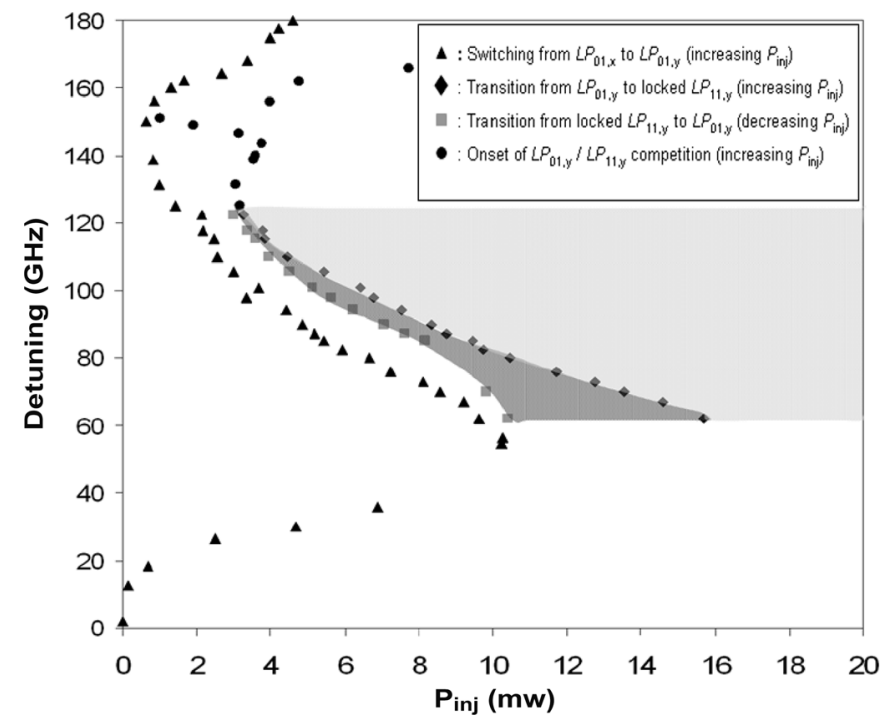

Fig. 8. Experimental mapping of PS and transverse modes competition in the plane detuning versus injection power $\left(P_{\text {inj }}\right)$. The bias current and the operating temperature are fixed at $2.105 \mathrm{~mA}$ and $20^{\circ} \mathrm{C}$, respectively.

Fig. 8, the mapping of the VCSEL subject to optical injection is presented for a very large positive detuning range, i.e., from 2 to $180 \mathrm{GHz}$. For a fixed detuning value, polarization-resolved dynamics as well as transverse mode competition are analyzed when the injection strength is scanned. If the injection strength is increased, and depending on the frequency detuning, different switching scenarios are resolved. A switching mechanism that involves the VCSEL fundamental orthogonal transverse modes, i.e., from the horizontal ( $x$-LP) to the vertical ( $y$-LP) mode, is observed for the whole frequency detuning range. The corresponding boundary is labeled by black triangles in Fig. 8. This boundary exhibits two minima for the switching power. A first minimum is located at a detuning of $2 \mathrm{GHz}$ for which PS is achieved at $7.1 \mu \mathrm{W}$. A second minimum for the switching power is found for a detuning of $150 \mathrm{GHz}$ and an injection power of $623.9 \mu \mathrm{W}$. It is worth mentioning that the second minimum is at much larger power than the one for a detuning of $2 \mathrm{GHz}$.

We analyze in more detail the transverse mode competition behavior for detunings ranging from 61 to $120 \mathrm{GHz}$ (see Fig. 8). With increasing the injection power we first observe PS between the $\mathrm{LP}_{01, x}$ and the $\mathrm{LP}_{01, y}$ modes (denoted by black triangles). When increasing further the injection power we observe injection locking of the $\mathrm{LP}_{11, y}$ mode - its frequency locks to the one of the ML, together with suppression of the fundamental transverse mode $\mathrm{LP}_{01, y}$. The corresponding injection locking boundary is denoted by black diamonds. Fig. 9 represents a sample of such a scenario for a detuning of $100 \mathrm{GHz}$ by using the experimental optical spectra. The experimental spectra shown in this paper have been recorded in the vertical polarization direction only. As the injection strength is increased, the VCSEL is initially frequency pushed but still emits a horizontal $x$-LP mode Fig. 9(a) . For a further increase in the injection strength, switching from horizontal $x$-LP to vertical $y$-LP fundamental mode is achieved, as shown by the sudden increase of the SL peak in vertical polarization direction in Fig. 9(b). By still increasing the injection strength, an abrupt injection locking of the 

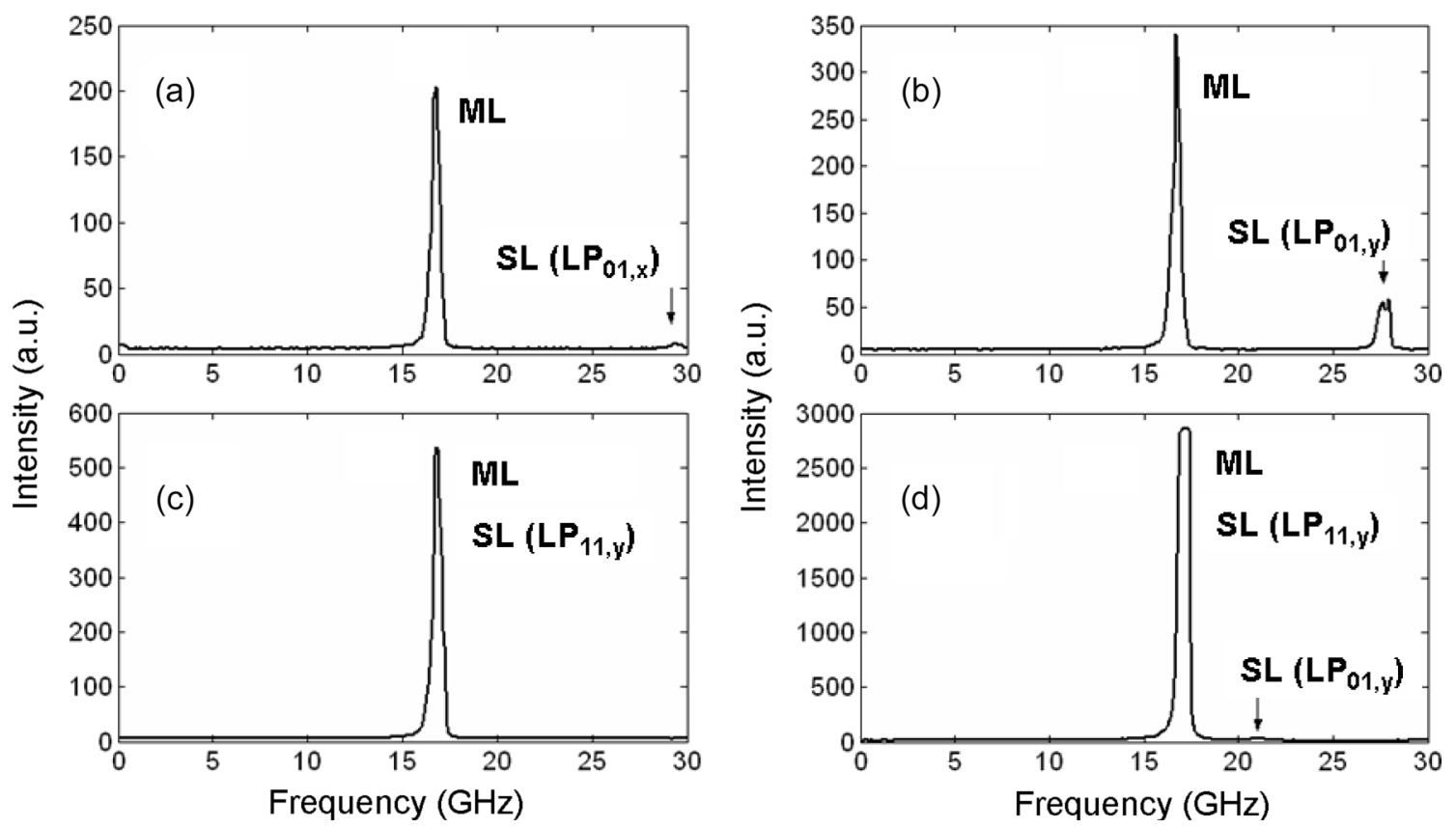

Fig. 9. Polarization mode competition when the detuning is fixed at $100 \mathrm{GHz}$. (a) VCSEL emits in horizontal before PS, $P_{\text {inj }}=3.26 \mathrm{~mW}$. (b) After PS to vertical polarization, $P_{\mathrm{inj}}=5.24 \mathrm{~mW}$. (c) Injection locking of the $\mathrm{LP}_{11, y}$ mode, $P_{\mathrm{inj}}=7.99 \mathrm{~mW}$. (d) Injection locking of the $\mathrm{LP}_{11, y}$ mode with relatively weak recovery of the VCSEL fundamental mode $P_{\text {inj }}=18 \mathrm{~mW}$.

first-order transverse mode to ML with suppression of the fundamental mode is resolved [see Fig. 9(c)]. It is worth mentioning that for a much higher injection power, a relatively weak emission of the fundamental $y$-LP mode can be observed with still the injection locked $\mathrm{LP}_{11, y}$ mode dominating (Fig. 9(d)). Bistability is observed if the injection power is decreased after injection locking of the $\mathrm{LP}_{11, y}$ mode is achieved, i.e., the VCSEL unlocks at an injection strength less than the one necessary to induce the locking regime (see the boundary labeled with light gray squares in Fig. 8). The width of the bistable region associated to the locking of the $\mathrm{LP}_{11, y}$ mode decreases as we increase the detuning as indicated by the zone with a dark gray shadding in Fig. 8.

For frequency detunings larger than $120 \mathrm{GHz}$, injection locking of the $\mathrm{LP}_{11, y}$ mode accompanied by suppression of the fundamental transverse mode $\mathrm{LP}_{01, y}$ is not observed anymore (see Fig. 10). Fig. 10(a) represents the situation for which the VCSEL is under optical injection but the injection strength is not sufficient to induce PS. By increasing the injection level PS from $x$-LP to $y$-LP fundamental modes is achieved Fig. 10(b). A further increase in the injection strength leads to a strong competition between the $\mathrm{LP}_{01, y}$ and $\mathrm{LP}_{11, y}$ modes. The onset of such a mode competition is shown on the mapping in Fig. 8 by black circles which correspond to the observation of a progressive decrease of the intensity at the SL frequency and a relatively strong increase of power at the ML frequency [see Fig. 10(c)]. Again, at a much stronger injection, a weak increase of the intensity at the SL frequency, i.e., a recovery of the $y$-LP fundamental mode, has been observed [Fig. 10(d) and the inset]. As shown in Fig. 8, the transverse mode competition appears at much lower injection power for a detuning of $150 \mathrm{GHz}$, which corresponds to the second minimum of the switching power. For larger positive detunings up to around $165 \mathrm{GHz}$, the mode competition is still resolved but at progressively increasing injection levels. Above this detuning range and as we increase the injection power, PS between the fundamental modes is still observed but afterwards the VCSEL keeps emitting an unlocked $y$-LP fundamental mode.

\section{DISCUSSION AND CONCLUSIONS}

We now discuss the similarities and the differences between the experimental and theoretical results presented in this work. The experimental light-current curve shows a switching from the higher to the lower frequency [see Fig. 7(a) at a current around $2 \mathrm{~mA}$ ] that does not appear in our theoretical results (see Fig. 1). That switching would also appear in our theoretical results if we would consider an additional physical mechanism with a significant influence on the polarization of the device, for instance, successive types I and II switchings have been observed when adding thermal [38] or polarization dependent gain/loss mechanisms [21] to SFM model. However, the absence of such switching in the theoretical results is not so important because the PS which we have been investigating is the second one, from the lower to the higher frequency polarization mode. In both, experiment and theory, this switching occurs within the fundamental mode of the device. Also in both cases small bumps in the $y$-polarization appear for currents smaller than the PS current due to the appearance of eliptically polarized states. Excitation of the first-order mode occurs at similar currents (at around five times the threshold current). A wide bistability region also appears in both cases in such a way that the switching from the $y$ - to the $x$-polarization when decreasing the current is also similar (at around two times the threshold current). We then conclude that the essential qualitative features of the experimental light current characteristics are well described by our model. 

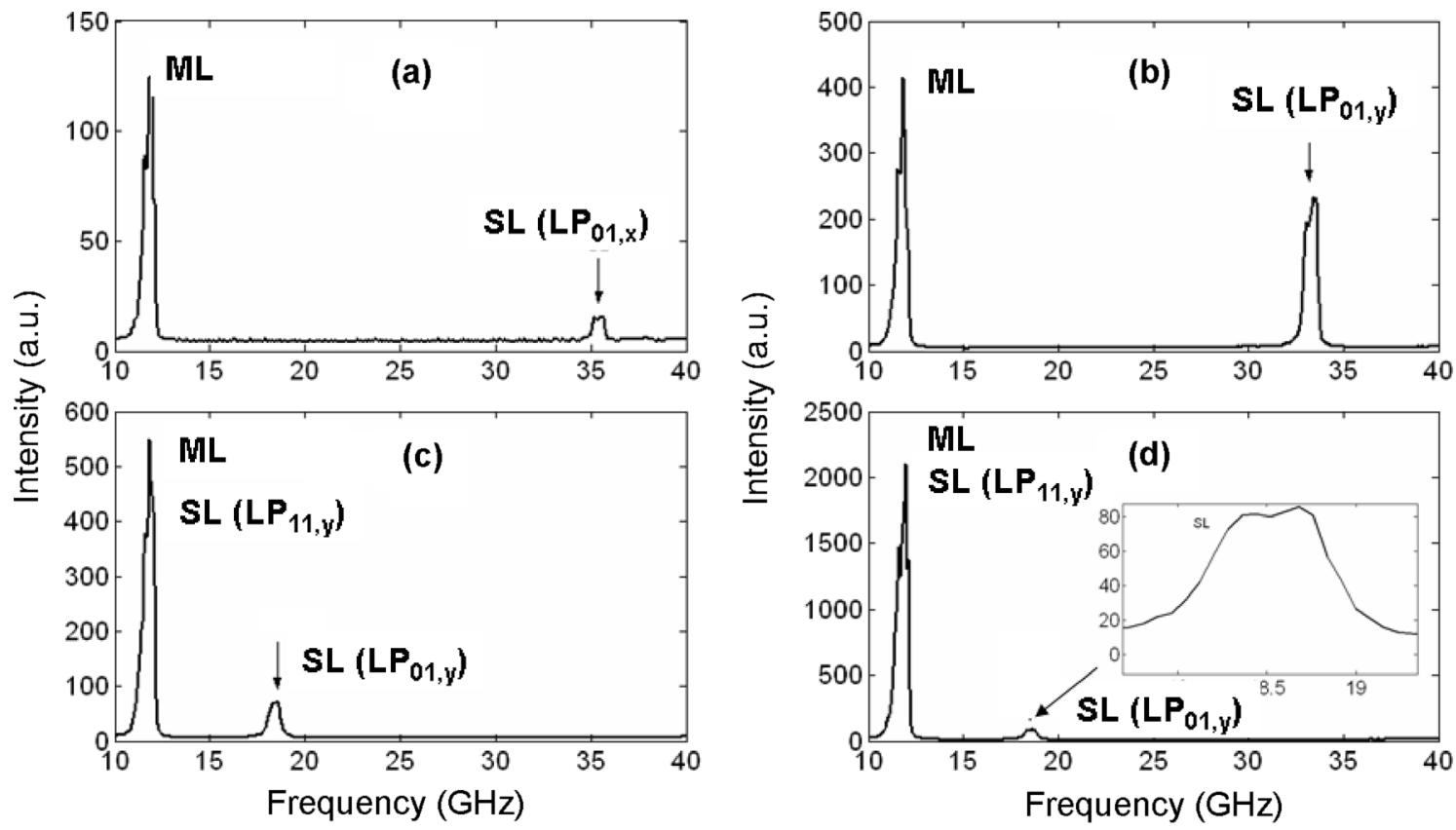

Fig. 10. Polarization mode competition when the detuning is fixed at $125 \mathrm{GHz}$. (a) VCSEL emits in horizontal before PS, $P_{\text {inj }}=0.72 \mathrm{~mW}$. (b) After PS to vertical polarization, $P_{\text {inj }}=2.23 \mathrm{~mW}$. (c) Injection locking of the $\mathrm{LP}_{11, y}$ mode to the ML after a progressive decrease of power at the SL frequency $P_{\mathrm{inj}}=6.97 \mathrm{~mW}$. (d) Injection locking of the $\mathrm{LP}_{11, y}$ mode with a relatively weak recovery of the VCSEL fundamental $\mathrm{LP}_{01, y}$ mode $P_{\text {inj }}=22.13 \mathrm{~mW}$.

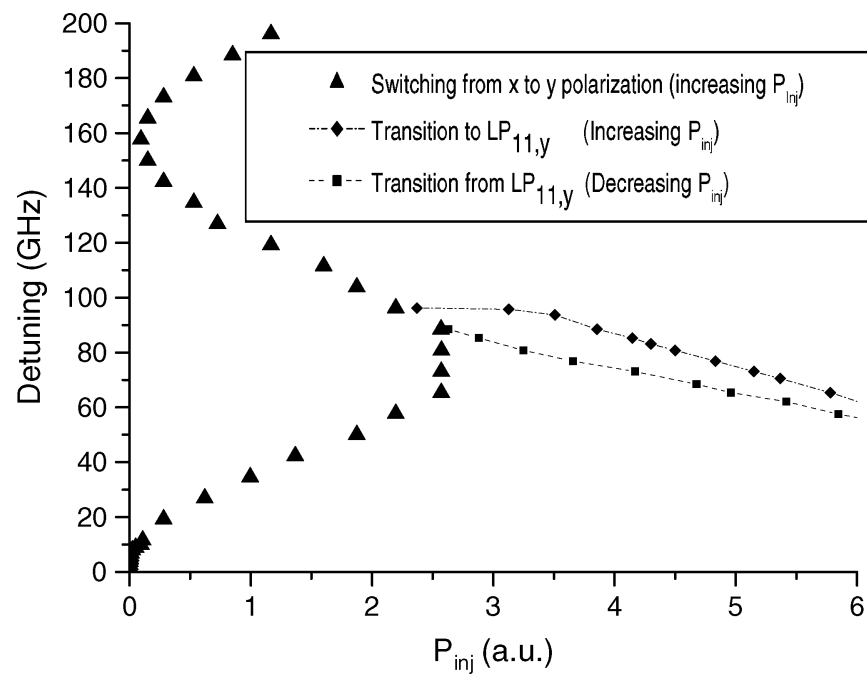

Fig. 11. Injection power required for PS in a VCSEL subject to orthogonal optical injection. Switching from $x$ - to $y$-polarization when increasing the injection power is shown with triangles. The injection power required for the $\mathrm{LP}_{11, y}$ modal power to reach an appreciable value, 0.5 , has been plotted if $P_{\text {inj }}$ is increased (diamonds) and if $P_{\mathrm{inj}}$ is decreased (squares). Parameters correspond to those in Fig. 2(a).

A comparison between the theoretical (Fig. 2) and the experimental (Fig. 8) mapping of the injection power required for PS shows that both theory and experiment feature a new minimum at a frequency detuning of around $150 \mathrm{GHz}$. A more detailed comparison is done in Fig. 11 where we have plotted the results from Fig. 2(a) in a linear horizontal axis. The injection power required for PS when increasing the injection power is plotted with black triangles. The experimental detuning frequencies at which the injection power is minimum or maximum are similar to the theoretical ones. As shown in Section III, the detuning frequency at which the new minimum occurs depends on the relative losses between the two transverse modes. The theoretical relative losses in Fig. 11 correspond to a VCSEL that becomes multimode at 4.7 times threshold. This result is consistent with the experimental result in which the first-order mode appears at five times threshold. The experimental $P_{\text {inj }}$ required for PS at the second minimum at $150 \mathrm{GHz}$ detuning, is $19.5 \mathrm{~dB}$ higher than the first one at $2 \mathrm{GHz}$ detuning, while the corresponding theoretical quantity is $23.8 \mathrm{~dB}$. Also, the experimental (theoretical) $P_{\text {inj }}$ required for PS at $150 \mathrm{GHz}$, is 16 (27) times smaller than the maximum one.

We find experimentally that, in the whole frequency detuning range we investigate, the PS involves the VCSEL fundamental orthogonal transverse modes, i.e., from the $\mathrm{LP}_{01, x}$ to the $\mathrm{LP}_{01, y}$ modes. However our theoretical results also unveil an additional possible scenario, in which a switching from the $\mathrm{LP}_{01, x}$ to the $\mathrm{LP}_{11, y}$ mode is observed when increasing the injection strength [see Fig. 3(e) and (f)]. Theoretically, we find that this second switching scenario appears for frequency detunings larger than $85 \mathrm{GHz}$ and for a value of the injection strength larger than that leading to a switching between the orthogonal fundamental transverse modes [compare Fig. 3(a) and (b) with Fig. 3(e) and (f)]. We find that this second switching scenario appears at smaller frequency detunings when increasing the $\kappa_{\mathrm{r}}$ parameter $\left(65 \mathrm{GHz}\right.$ when $\left.\kappa_{\mathrm{r}}=1.4\right)$. In this way, a wider range of frequency detunings over which the $\mathrm{LP}_{01, x}$ switches to the $\mathrm{LP}_{01, y}$ modes would be obtained if the $\kappa_{r}$ parameter is decreased. Whether it is possible to observe this second switching scenario in experiment remains an interesting question for future investigations. We also find experimentally that the $\mathrm{LP}_{11, y}$ mode appears locked to the injection. This is also the case for our theoretical results since our optical spectra 
indicate locking of the $\mathrm{LP}_{11, y}$ mode can be achieved for all the considered frequency detuning range. Bistability in PS has been found in the experiment and theory and is demonstrated in Fig. 11 where the injection power required for the $\mathrm{LP}_{11, y}$ modal power to reach an appreciable value has been plotted when increasing (diamonds) and when decreasing (squares) the injection power. Theoretically, this bistability can be for the fundamental mode only or for both transverse modes. In such a way, our numerical results complement the experiment, which can not distinguish for the contribution to the PS and for the hysteresis of the two transverse modes separately.

To summarize, we have performed theoretical and experimental investigations of VCSELs subject to orthogonal optical injection as a function of the injection strength and of the frequency detuning between the master and the SLs. These investigations have extended previous experimental work over larger frequency detunings. Within this extended range, we have found a new minimum of the injection power needed to obtain PS that appears at a frequency detuning that is near the frequency difference between the fundamental and the first-order transverse modes of the solitary VCSEL. We have found that both the depth and the frequency position of this minimum increase when considering lasers that become multitransverse mode at lower injection currents. Our theoretical and experimental results have also shown that bistability in PS is obtained for all the frequency detuning range. Such a bistability is obtained for the fundamental mode only or for both transverse modes, depending on the value of the detuning. The theoretical and experimental optical spectra have shown that the first-order transverse mode appears locked to the external injection. Our theoretical model have captured most of the fundamental features of the experiment.

\section{ACKNOWLEDGMENT}

The first author, A.Valle, would like to thank M. S. Torre and L. Pesquera for their preliminary work in the model used in this paper.

\section{REFERENCES}

[1] R. Lang, "Injection locking properties of a semiconductor laser," IEEE J. Quantum Electron., vol. QE-18, no. 6, pp. 976-983, Jun. 1982.

[2] G. H. M. Van Tartwijk and D. Lenstra, "Semiconductor lasers with optical injection and feedback," Quantum Semiclass. Opt., vol. 7, no. 2, pp. 87-143, 1995.

[3] P. Gallion, H. Nakajima, G. Debarge, and C. Chabran, "Contribution of spontaneous emission to the linewidth of an injection-locked semiconductor laser," Electron. Lett., vol. 22, pp. 626-628, 1995.

[4] K. Iwashita and K. Nakagawa, "Suppression of mode partition noise by laser diode light injection," IEEE. J. Quantum Electron., vol. QE-18, no. 10 , pp. 1669-1674, Oct. 1982.

[5] N. A. Olsson, H. Temkin, R. A. Logan, L. F. Johnson, G. J. Dolan, J. P. Van der Ziel, and J. C. Campbell, "Chirp-free transmission over $82.5 \mathrm{~km}$ of single mode fibers at $2 \mathrm{~Gb} / \mathrm{s}$ with injection locked DFB semiconductor lasers," J. Lightw. Technol., vol. 3, no. 2, pp. 63-67, Feb. 1985.

[6] X. Meng, T. Chau, and M. C. Wu, "Experimental demonstration of modulation bandwidth enhacement in distributed feedback lasers with external light injection," Electron. Lett., vol. 34, no. 21, pp. 2031-2032, 1998.

[7] B. K. Mathason and P. J. Delfyett, "Pulsed injection locking dynamics of passively mode-locked external-cavity semiconductor laser systems for all-optical clock recovery," J. Lightw. Technol., vol. 18, no. 8, pp. 1111-1120, Aug. 2000.

[8] S. Yamashita and D. Matsumoto, "Waveform reshaping based on injection locking of a distributed-feedback semiconductor laser," IEEE Photon. Technol. Lett., vol. 12, no. 10, pp. 1388-1390, Oct. 2000.
[9] L. Goldberg, H. F. Taylor, J. F. Weller, and D. M. Bloom, "Microwave signal generation with injection-locked laser diodes," Electron. Lett., vol. 19, no. 13, pp. 491-493, 1983.

[10] R. P. Braun, G. Grosskopf, D. Rohde, and F. Schmidt, "Low phasenoise millimeter-wave generation at $64 \mathrm{GHz}$ and data transmission using optical sideband injection locking," IEEE Photon. Technol. Lett., vol. 10, no. 5, pp. 728-730, May 1998.

[11] A. C. Bordonalli, C. Walton, and A. J. Seeds, "High-performance phase locking of wide linewidth semiconductor lasers by combined use of optical injection locking and optical phase-lock loop," J. Lightw. Technol., vol. 17, no. 2, pp. 328-342, Feb. 1999.

[12] X. J. Meng, T. Chau, and M. C. Wu, "Improved intrinsic dynamic distortions in directly modulated semiconductor lasers by optical injection locking," IEEE Trans. Microw. Theory Tech., vol. 47, no. 7, pp. 1172-1176, Jul. 1999.

[13] C. J. Chang-Hasnain, J. P. Harbison, G. Hasnain, A. C. von Lehmen, L. T. Florez, and N. G. Stoffel, "Dynamic, polarization and transverse mode characteristics of vertical cavity surface emitting lasers," IEEE J. Quantum Electron., vol. 27, no. 6, pp. 1402-1409, Jun. 1991.

[14] D. Vakhshoori, J. D. Wynn, G. J. Zydzik, M. Asom, K. Kojima, R. E. Leibenguth, and R. A. Morgan, "Top-surface emitting lasers with $1.9 \mathrm{~V}$ threshold voltage and the effect of spatial hole burning on their transverse mode operation and efficiencies," Appl. Phys. Lett., vol. 62, no. 13 , pp. 1448-1450, 1993.

[15] A. Valle, J. Sarma, and K. A. Shore, "Spatial hole burning effects on the dynamics of vertical-cavity surface-emitting laser diodes," IEEE J. Quantum Electron., vol. 31, no. 8, pp. 1423-1431, Aug. 1995.

[16] M. San Miguel, Q. Feng, and J. V. Moloney, "Light-polarization dynamics in surface-emitting semiconductor lasers," Phys. Rev. A, vol. 52, no. 2, pp. 1728-1739, 1995.

[17] J. M. Martín-Regalado, F. Prati, M. San Miguel, and N. B. Abraham, "Polarization properties of vertical cavity surface-emitting lasers," IEEE J. Quantum Electron., vol. 33, no. 5, pp. 765-783, May 1997.

[18] K. D. Choquette, R. P. Schneider, K. L. Lear, and R. E. Leibenguth, "Gain-dependent polarization properties of vertical-cavity lasers," IEEE J. Sel. Topics Quantum Electron., vol. 1, no. 2, pp. 661-666, Mar./Apr. 1995.

[19] K. Panajotov, B. Ryvkin, J. Danckaert, M. Peeters, H. Thienpont, and I. Veretennicoff, "Polarization switching in VCSELs due to thermal lensing," IEEE Photon. Technol. Lett., vol. 10, no. 1, pp. 6-8, Jan. 1998.

[20] A. Valle, L. Pesquera, and K. A. Shore, "Polarization behavior of birefringent multitransverse mode vertical-cavity surface-emitting lasers," IEEE Photon. Technol. Lett, vol. 9, no. 5, pp. 557-559, May 1997.

[21] B. Ryvkin, K. Panajotov, A. Georgievski, J. Danckaert, M. Peeters, G. Verschaffelt, H. Thienpont, and I. Veretennicoff, "Effect of photonenergy-dependent loss and gain mechanisms on polarization switching in vertical-cavity surface-emitting lasers," J. Opt. Soc. Amer. B, vol. 16, pp. 2106-2113, 1999.

[22] C. H. Chang, L. Chrostowski, and C. J. Chang-Hasnain, "Injection locking of VCSELs," IEEE J. Sel. Topics Quantum Electron., vol. 9, no. 5, pp. 1386-1393, Sep./Oct. 2003.

[23] Y. Onishi, F. Koyama, N. Nishiyama, C. Caneau, and C. E. Zah, "Nonlinear optical optical input-output characteristics of 1.55 microns injection-locked vertical-cavity surface-emitting lasers," Appl. Phys. Lett., vol. 84, no. 17, pp. 3247-3249, 2004.

[24] K. Panajotov, F. Berghmans, M. Peeters, G. Verschaffelt, J. Danckaert, I. Veretennicoff, and H. Thienpont, "Data transparent reconfigurable optical interconnections using polarization switching in VCSELs induced by optical injection," IEEE Photon. Technol. Lett., vol. 11, no. 8, pp. 985-987, Aug. 1999.

[25] Y. Hong, P. S. Spencer, P. Rees, and K. A. Shore, "Optical injection dynamics of two-mode vertical-cavity surface-emitting semiconductor lasers," IEEE J. Quantum Electron., vol. 38, no. 3, pp. 274-278, 2002.

[26] Y. Onishi, N. Nishiyama, C. Caneau, F. Koyama, and C. E. Zah, "Dynamic behavior of an all-optical inverter using transverse-mode switching in 1.55 microns vertical-cavity surface-emitting lasers," IEEE Photon. Technol. Lett., vol. 16, no. 5, pp. 1236-1238, May 2004.

[27] H. Li, T. L. Lucas, J. G. McInerney, M. W. Wright, and R. A. Morgan, "Injection locking dynamics of vertical cavity semiconductor lasers under conventional and phase conjugate injection," IEEE J. Quantum Electron., vol. 32, no. 2, pp. 227-235, Feb. 1996.

[28] Y. Hong, P. S. Spencer, S. Bandyopadhyay, P. Rees, and K. A. Shore, "Polarization-resolved chaos and instabilities in a vertical-cavity surface-emitting laser subject to optical injection," Opt. Commun., vol. 216, pp. 185-189, 2003. 
[29] Z. G. Pan, S. Jiang, M. Dagenais, R. A. Morgan, K. Kojima, M. T. Asom, and R. E. Leibenguth, "Optical injection induced polarization bistability in vertical-cavity surface-emitting lasers," Appl. Phys. Lett., vol. 63, pp. 2999-3001, 1993.

[30] J. Buesa, I. Gatare, K. Panajotov, H. Thienpont, and M. Sciamanna, "Mapping of the dynamics induced by orthogonal optical injection in vertical-cavity surface-emitting laser," IEEE J. Quantum Electron., vol. 42, no. 2, pp. 198-207, Feb. 2006.

[31] I. Gatare, M. Sciamanna, J. Buesa, H. Thienpont, and K. Panajotov, "Nonlinear dynamics accompanying polarization switching in verticalcavity surface-emitting lasers with orthogonal optical injection," Appl. Phys. Lett., vol. 88, 2006, 101106.

[32] M. Sciamanna and K. Panajotov, "Two-mode injection locking in vertical-cavity surface-emitting lasers," Opt. Lett., vol. 30, pp. 2903-2905, 2005.

[33] — - "Route to polarization switching induced by optical-injection in vertical-cavity surface-emitting lasers," Phys. Rev. A, vol. 73, no. 2, 2006, 023811 .

[34] J. M. Martín-Regalado, S. Balle, M. San Miguel, A. Valle, and L. Pesquera, "Polarization and transverse-mode selection in quantum-well vertical-cavity surface-emitting lasers: Index- and gain-guided devices," Quantum Semiclass. Opt., vol. 9, pp. 713-736, 1997.

[35] M. S. Torre, A. Valle, and L. Pesquera, "Polarization and transverse mode behavior of VCSELs under optical injection," Opt. Quantum Electron., vol. 38, pp. 445-465, 2006.

[36] A. Valle, K. A. Shore, and L. Pesquera, "Polarization selection in birefringent vertical-cavity surface emitting lasers," J. Lightw. Technol, vol. 14, no. 9, pp. 2062-2068, Sep. 1996.

[37] J. Mulet and S. Balle, "Transverse mode dynamics in vertical-cavity surface-emitting lasers: Spatiotemporal versus modal expansion descriptions," Phys. Rev. A, vol. 66, no. 5, 2002, 053802.

[38] S. Balle, E. Tolkachova, M. San Miguel, J. R. Tredicce, J. Martín-Regalado, and A. Gahl, "Mechanisms of polarization switching in singletransverse mode vertical-cavity surface-emitting lasers: Thermal shift and nonlinear semiconductor dynamics," Opt. Lett., vol. 24, no. 16, pp. 1121-1123, 1999

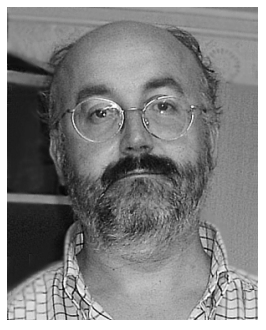

Angel Valle was born in Reinosa, Cantabria, Spain, in 1965. He received the M.Sc. and Ph.D. degree in physics from the Universidad de Cantabria, Spain, in 1988 and 1993, respectively.

During 1994 and 1995, he was a Postdoctoral Fellow at the School of Electronic and Electrical Engineering at the University of Bath, Bath, U.K. In 1996, he joined the Instituto de Física de Cantabria (CSIC-UC). Since 1998, he has been a Lecturer at the Departamento de Física Moderna, University of Cantabria, Cantabria, Spain. His research interests are in the areas of vertical-cavity surface-emitting lasers, noise, and nonlinear dynamics of semiconductor lasers.

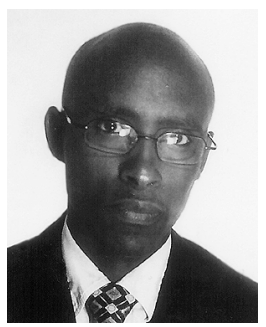

Ignace Gatare received the M.S. degree in electrical engineering from the Faculté Polytechnique de Mons, Mons, Belgium, in 2004. He is currently working toward the Ph.D. degree jointly at the Vrije Universiteit Brussel (VUB), Belgium, and the Ecole Supérieure d'Electricité (Supélec), Metz, France.

His research interests include polarization switching dynamics induced by optical injection in VCSEL and chaos synchronization of coupled VCSELS.

Mr. Gatare has been awarded with the faculty Prize of Best Master Thesis in Telecommunications granted by SEE telecom, Belgium (2004). He is a student member of the IEEE LEOS.

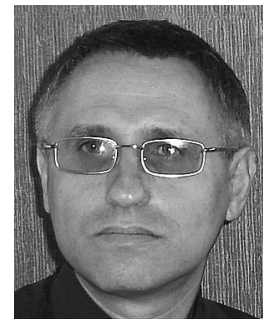

Krassimir Panajotov received the M.S., Ph.D., and D.Sc. degrees in physics in from Sofia University, Sofia, Bulgaria, in 1982, 1988, and 2002, respectively.

Since 1982, he has been with the Institute of Solid State Physics (ISSP), Bulgarian Academy of Sciences, Sofia, Bulgaria, where he is currently a Professor. Since 2001, he has led the group on Micro- and Nano-Photonics at ISSP. During 1996-1998, he was a Guest Researcher at the Vrije Universiteit Brussels (VUB), Brussels, Belgium, and since 2000, he has been a Guest Professor. His research activities are in thin film and fiber optics, nonlinear optics, semiconductor lasers, and nonlinear laser dynamics and photonic crystal devices. In these fields, he holds more than 80 SCI-stated journal papers and more than 90 publications in international conference proceedings. He was an invited speaker at a number of international conferences and serves as a referee in several journals. He has led several projects on national level in Bulgaria, as well as international bilateral and European projects. During 1999-2002, he served as a National (Bulgarian) coordinator of the Cost 268 initiative, and is currently National coordinator for two ongoing COST actions: COST 288 and COST P11. He co-chaired the PHASE international workshop (PHysics and Applications of SEmiconductor LASERs) in Supélec, Metz, France, in March 2005 and acted as a Guest Editor of a Special Issue of Optical and Quantum Electronics related to the PHASE workshop. He also co-chaired the PPCM international workshops (Physics of Photonic Crystals and Metamaterials) in VUB, Brussels, in June, 2006.

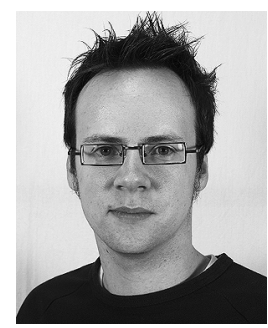

Marc Sciamanna received the M.S. degree in electrical engineering and the Ph.D. degree as a Research Fellow of the "Fonds National de la Recherche Scientifique" (FNRS), from the Faculté Polytechnique de Mons, Mons, Belgium, in 2000. His Ph.D. dissertation is entitled "Nonlinear dynamics and polarization properties of externally driven semiconductor lasers."

He is currently a permanent Researcher, Lecturer, and Faculty Staff Member of Ecole Supérieure d'Electricité (SUPELEC) and Laboratoire Matériaux Optiques, Photonique et Systèmes (LMOPS), Centre National de la Recherche Scientifique (CNRS), Metz (France). His research interests include the physics and nonlinear dynamics of semiconductor lasers, the polarization properties of VCSELs, the study of dynamical instabilities related to optical feedback, optical injection, or large current modulation, synchronization and chaotic encryption using laser diodes. He is author of about 50 research papers in international journals and conference proceedings. He has given several invited talks in international conferences and has worked as a scientific program committee member in SPIE Photonics Europe 2006. He organized and cochaired the PHASE international workshop (PHysics and Applications of SEmiconductor LASERs), Metz, France, in March 2005 and acted as a Guest Editor of a special issue of Optical and Quantum Electronics related to the PHASE workshop. He acted as a Board Member of the IEEE/LEOS Benelux Chapter, co-founded the IEEE/LEOS Benelux Student Chapter, and co-chaired the IEEE/LEOS Workshop on Low-Cost Photonics (Mons, Belgium, June, 2003). $\mathrm{He}$ is Vice-Chairman of working group 2 "Physics of Devices" in European Action COST 288

Dr. Sciamanna has been awarded with the Prize for Best Engineer in Electricity from Faculté Polytechnique de Mons (2000), the OSA-Newfocus Student Travel Grant Award (2002), the IEEE/LEOS Graduate Student Fellowship Award (2002), and the SPIE F-MADE Scholarship Award in Optical Engineering (2003). 